\title{
Improved Heuristics for Multi-Agent Path Finding with Conflict-Based Search
}

\author{
Jiaoyang Li ${ }^{1}$, Ariel Felner ${ }^{2}$, Eli Boyarski ${ }^{2}$, Hang Ma ${ }^{1}$ and Sven Koenig ${ }^{1}$ \\ ${ }^{1}$ University of Southern California \\ ${ }^{2}$ Ben Gurion University of the Negev \\ jiaoyanl@usc.edu, felner@bgu.ac.il, boyarske@post.bgu.ac.il, \{hangma, skoenig $\} @ u s c . e d u$
}

\begin{abstract}
Conflict-Based Search (CBS) and its enhancements are among the strongest algorithms for Multi-Agent Path Finding. Recent work introduced an admissible heuristic to guide the high-level search of CBS. In this work, we prove the limitation of this heuristic, as it is based on cardinal conflicts only. We then introduce two new admissible heuristics by reasoning about the pairwise dependencies between agents. Empirically, CBS with either new heuristic significantly improves the success rate over CBS with the recent heuristic and reduces the number of expanded nodes and runtime by up to a factor of 50 .
\end{abstract}

\section{Introduction}

Multi-Agent Path Finding (MAPF) is the problem of finding a set of collision-free paths for a given set of agents on a given graph. Although MAPF is NP-hard to solve optimally [Yu and LaValle, 2013b], many optimal MAPF algorithms have been developed in recent years, including reduction-based algorithms [Yu and LaValle, 2013a; Erdem et al., 2013; Surynek et al., 2016; Barták et al., 2017], A*based algorithms [Standley, 2010; Wagner and Choset, 2011; Goldenberg et al., 2014] and dedicated search-based algorithms [Sharon et al., 2013; Sharon et al., 2015].

Conflict-Based Search (CBS) [Sharon et al., 2015] is a popular two-level search-based MAPF algorithm which resolves collisions by adding constraints at a high level and computing paths consistent with those constraints at a low level. It is widely used in many real-world applications, such as warehouse robots [Ma et al., 2017a; Hönig et al., 2019], quadrotor swarms [Hönig et al., 2018] and computer game characters [Ma et al., 2017b].

A number of enhancements to CBS have been introduced [Barer et al., 2014; Boyarski et al., 2015; Cohen et al., 2016; Cohen et al., 2018; Li et al., 2019a; Li et al., 2019b; Gange et al., 2019]. CBSH [Felner et al., 2018] was the first work that introduced an admissible heuristic (called here CG) for the high-level search of CBS by reasoning about a special type of collisions in the current solution (i.e., the paths in the current high-level node). In this paper, we further develop this direction. We first prove that CG can offer only a limited amount of information. We then introduce two new admissible heuristics, DG and WDG, by considering potential collisions in future solutions (i.e., the paths in the descendant high-level nodes) and reasoning about the pairwise dependencies between agents. WDG strictly dominates DG, which in turn strictly dominates CG. Empirically, the runtime overhead of calculating the new heuristics is reasonable, and WDG improves the success rate of CBS significantly compared to $C G$ and reduces the number of expanded nodes and runtime by up to a factor of 50 .

\section{Background}

\subsection{Problem Definition}

The Multi-Agent Path Finding (MAPF) problem is specified by an undirected unweighted graph $G=(V, E)$ and a set of $k$ agents $\left\{a_{1} \ldots a_{k}\right\}$, where $a_{i}$ has start vertex $s_{i} \in V$ and goal vertex $g_{i} \in V$. Time is discretized into timesteps. Between successive timesteps, every agent can either move to an adjacent vertex or wait at its current vertex. Both move and wait actions have unit cost unless the agent terminally waits at its goal vertex, which has zero cost. A path of $a_{i}$ is a sequence of move and wait actions that lead $a_{i}$ from $s_{i}$ to $g_{i}$. A tuple $\left\langle a_{i}, a_{j}, v, t\right\rangle$ is a vertex conflict iff $a_{i}$ and $a_{j}$ are at the same vertex $v$ at timestep $t$, and a tuple $\left\langle a_{i}, a_{j}, u, v, t\right\rangle$ is an edge conflict iff $a_{i}$ and $a_{j}$ traverse the same edge $(u, v)$ in opposite directions between timesteps $t$ and $t+1$. The objective that we focus on in this paper is to find a set of conflict-free paths which move all agents from their start vertices to their goal vertices while minimizing the sum of the costs of these paths.

\subsection{Conflict-Based Search (CBS)}

CBS has two levels. The high level of CBS searches the binary constraint tree (CT) in a best-first manner according to the costs of the CT nodes. Each CT node $N$ contains:

(1) a set of constraints $N$.constraints, where a constraint is either a vertex constraint $\left\langle a_{i}, v, t\right\rangle$ that prohibits agent $a_{i}$ from being at vertex $v$ at timestep $t$ or an edge constraint $\left\langle a_{i}, u, v, t\right\rangle$ that prohibits agent $a_{i}$ from moving from vertex $u$ to vertex $v$ between timesteps $t$ and $t+1$;

(2) a solution N.solution, that consists of a set of $k$ cost-minimal paths, one for each agent, that satisfy N.constraints; and

(3) a cost N.cost, that is equal to the sum of the costs of the paths in N.solution. 
The root CT node contains an empty set of constraints.

When CBS chooses a CT node $N$ for expansion, it checks for conflicts in N.solution. If there are none, CBS terminates and returns N.solution. Otherwise, CBS chooses one of the conflicts (by default, arbitrarily) and resolves it by splitting $N$ into two child CT nodes. In each child CT node, one agent from the conflict is prohibited from using the contested vertex or edge by way of an additional constraint. The path of this agent then no longer satisfies the constraints of the child CT node and must be replanned by a low-level search (e.g., a time-space $A^{*}$ search [Silver, 2005]). All other paths remain unchanged. If the low-level search cannot find any path that satisfies the constraints, this child CT node does not have any solution and therefore is pruned. With two child CT nodes per conflict, CBS guarantees optimality by exploring both ways of resolving each conflict.

\subsection{Improved CBS (ICBS)}

CBS arbitrarily chooses conflicts to split on. However, poor choices can substantially increase the size of its CT and thus its runtime. Improved CBS (ICBS) [Boyarski et al., 2015] addresses this issue by prioritizing conflicts at each CT node $N$. It classifies conflicts into three types. A conflict is cardinal iff, when CBS uses it to split $N$, the cost of each of the two resulting child CT nodes is larger than $N$.cost. (i.e., a conflict is cardinal iff all shortest paths of the two conflicting agents traverse the conflicting vertex/edge at the conflicting timestep). It is semi-cardinal iff the cost of one child CT node is larger than N.cost, but the cost of the other child CT node is equal to N.cost. Finally, it is non-cardinal iff the cost of each of the two child nodes is equal to $N$.cost. ICBS must first choose a cardinal conflict (if one exists) when splitting $N$. For example, in Figure 1(left), the conflict $\left\langle a_{1}, a_{2}, B 2,1\right\rangle$ at the root CT node is non-cardinal as both agents have bypasses that reach their goal vertices at timestep 4 without being at $B 2$ at timestep 1. However, if cells $C 1$ and $A 3$ are blocked, the conflict becomes cardinal because, when $a_{1}$ or $a_{2}$ is prohibited from being at $B 2$ at timestep 1, it has to wait at its start vertex for 1 timestep and thus reaches its goal vertex only at timestep 5.

ICBS uses MDDs to classify conflicts. A Multi-Valued Decision Diagram (MDD) [Sharon et al., 2013] for $a_{i}$ at $N$ is a directed acyclic graph that consists of all cost-minimal paths of $a_{i}$ from $s_{i}$ to $g_{i}$ that satisfy $N$.constraints. Nodes at depth $t$ of the MDD for $a_{i}$ correspond to all vertices where $a_{i}$ can be at timestep $t$ along one of its cost-minimal paths. A conflict between $a_{i}$ and $a_{j}$ at timestep $t$ is cardinal iff the contested vertex (or edge) is the only vertex (or edge) at level $t$ of the MDDs for both agents. Figure 1(middle) shows the MDDs for $a_{1}$ and $a_{2}$ at the root CT node, respectively. Since both MDDs have 2 nodes at timestep 1 , the conflict $\left\langle a_{1}, a_{2}, B 2,1\right\rangle$ is non-cardinal.

\subsection{CBSH}

The high level of CBS always chooses to expand the CT node $N$ with the smallest N.cost. CBSH [Felner et al., 2018] speeds up the high-level search through the addition of an admissible heuristic. The idea is simple: If N.solution contains one cardinal conflict, then an $h$-value of 1 is admissible

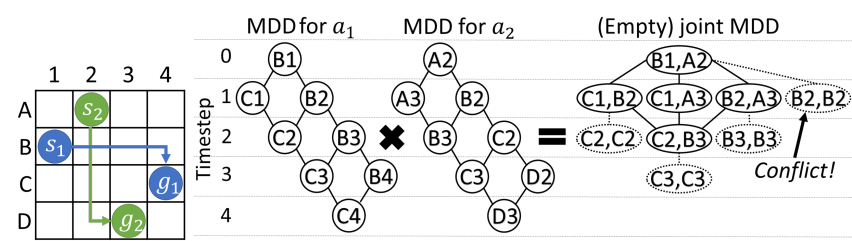

Figure 1: A MAPF instance on a 4-neighbor grid with the corresponding MDDs and joint MDD.

for $N$ because the cost of any of its descendant CT nodes with a conflict-free solution is at least $N$.cost +1 . If $N$.solution contains multiple cardinal conflicts, then CBSH builds a conflict graph, whose vertices represent agents and edges represent cardinal conflicts in N.solution. The cost of the path of at least one agent from each cardinal conflict has to increase by at least 1 . Thus, the size of a minimum vertex cover (MVC) of the conflict graph (i.e., a set of vertices such that each edge is incident on at least one vertex in the set) is an admissible $h$-value for $N$. We refer to this heuristic as the CG heuristic.

\section{Limitation of the CG Heuristic}

We have just seen that an $h$-value of 1 for a cardinal conflict is admissible. However, is it possible to find a larger $h$-value for a cardinal conflict? The following theorem answers the question. The proof of this theorem is given in the appendix.

Theorem 1. Suppose that CBS chooses to resolve a conflict between $a_{i}$ and $a_{j}$ at timestep $t$ at a CT node $N$ and both child CT nodes of $N, N_{1}$ (with an additional constraint imposed on $a_{i}$ ) and $N_{2}$ (with an additional constraint imposed on $a_{j}$ ) have solutions. If the conflict occurs after one of the agents, say $a_{i}$, reaches its goal vertex (i.e., $t \geq \mu_{i}$, where $\mu_{i}$ is the cost of the path of $a_{i}$ in N.solution), then $N_{1}$.cost $=$ $N$. cost $+t+1-\mu_{i}$ and $N_{2}$.cost $\in\{N$.cost,$N$.cost +1$\}$. Otherwise (i.e., it occurs before both agents reach their goal vertices), $N_{1}$.cost, $N_{2}$.cost $\in\{N$.cost, $N$.cost +1$\}$.

Therefore, if both child CT nodes of $N$ have solutions (which is always true in practice), a conflict can be regarded as an admissible $h$-value of at most 1 . Then the size of the MVC is the best admissible heuristic for $N$ that can be obtained from the conflict graph. So, if we want to obtain better heuristics, new directions need to be explored.

\section{The DG Heuristic}

The CG heuristic considers only cardinal conflicts in N.solution. To improve on that we also need to consider conflicts in future solutions, i.e., solutions of $N$ 's descendant CT nodes. For example, in Figure 1(left), if CBS resolves the non-cardinal conflict $\left\langle a_{1}, a_{2}, B 2,1\right\rangle$ by adding a constraint for one of the agents, a new conflict will occur no matter what new cost-minimal path the agent picks. In fact, any two costminimal paths of the two agents conflict in one of the 4 cells in the middle (B2,B3,C2,C3). Therefore, an $h$-value of 1 is admissible here. This is not captured by CG because the conflicts are initially non-cardinal. Inspired by this example, we generalize the conflict graph described above to a pairwise dependency graph, whose edges reflect that all cost-minimal paths of the corresponding two agents have conflicts. 


\subsection{Pairwise Dependency $\mathbf{G r a p h}_{\mathbf{G}_{\mathbf{D}}}$}

Formally, we define a pairwise dependency graph $G_{D}=$ $\left(V_{D}, E_{D}\right)$ for each $\mathrm{CT}$ node $N$. Each agent $a_{i}$ induces a vertex $v_{i} \in V_{D}$. An edge $\left(v_{i}, v_{j}\right) \in E_{D}$ iff $a_{i}$ and $a_{j}$ are dependent, i.e., all their cost-minimal paths that satisfy N.constraints have conflicts. Similarly to the conflict graph, for each edge $\left(v_{i}, v_{j}\right) \in E_{D}$, the cost of the path of at least one agent, $a_{i}$ or $a_{j}$, has to increase by at least 1 . Hence, the size of the MVC of $G_{D}$ is an admissible $h$-value for $N$. We refer to this heuristic as the DG heuristic. DG strictly dominates CG because the conflict graph is a sub-graph of $G_{D}$. We use the same algorithm as in [Felner et al., 2018] to determine an MVC. Its complexity is $O\left(2^{q}\left|V_{D}\right|\right)$, where $q$ is the size of the MVC.

\subsection{Constructing $\mathbf{G}_{\mathrm{D}}$}

To construct $G_{D}$ for $N$, we need to analyze the dependencies between every pair of agents. Let $\mu_{i}$ denote the cost of the path of $a_{i}$ in N.solution. We first classify all pairs of agents into three categories based on their conflicts in N.solution:

(1) The two agents do not have any conflicts.

(2) They have at least one cardinal conflict.

(3) They have only semi-cardinal or non-cardinal conflicts.

If $a_{i}$ and $a_{j}$ are in Category (1), they are independent as their paths in N.solution are conflict-free. Hence, $\left(v_{i}, v_{j}\right) \notin E_{D}$. If they are in Category (2), by the definition of cardinal conflicts, they are surely dependent. Hence, $\left(v_{i}, v_{j}\right) \in E_{D}$. If they are in Category (3), we do not know whether they are dependent or independent. To provide an answer, we try to merge the MDDs for the two agents into a joint MDD using the method described in [Sharon et al., 2013]. The two agents are dependent iff their joint MDD is empty. Details of the merging are given in Section 4.3.

Since each CT node has an additional constraint imposed on only one agent, we only need to look at the dependencies between this agent and all other agents and can copy the edges for the other pairs of agents from the $G_{D}$ for the parent $\mathrm{CT}$ node. Of course, at the root CT node, we still need to look at the dependencies for all pairs of agents. CG already build MDDs to classify conflicts. So, for DG, we get these MDDs for free. The only overhead of DG over CG comes from merging the MDDs.

\subsection{Merging the MDDs}

The joint MDD of the MDDs for $a_{i}$ and $a_{j}$ at $N$ consists of all combinations of cost-minimal conflict-free paths of $a_{i}$ and $a_{j}$ that satisfy N.constraints. Nodes at depth $t$ of the joint MDD correspond to all joint states where $a_{i}$ and $a_{j}$ can be at timestep $t$ along such a pair of paths without conflicts. Let $\mu_{k}$ $(k=i, j)$ denote the depth of the MDD for $a_{k}$. If $\mu_{i} \neq \mu_{j}$, a path of $\left|\mu_{i}-\mu_{j}\right|$ dummy goal vertices is added to the sink node of the shallower MDD (representing the agent sitting at its goal vertex) so that both MDDs have the same depth. The joint MDD is built level by level. The merging procedure starts at the joint state $\left(s_{i}, s_{j}\right)$ at level 0 . Suppose that we already have a joint state $\left(v_{i}, v_{j}\right)$ at level $t$ and want to add its child nodes at level $t+1$. Each pair in the cross product of the

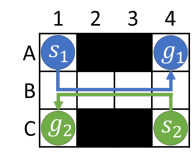

Figure 2: A MAPF instance on a 4-neighbor grid with $\Delta_{12}=4$.

child nodes of $v_{i}$ at level $t$ in the MDD for $a_{i}$ and the child nodes of $v_{j}$ at level $t$ in the MDD for $a_{j}$ should be examined. Only conflict-free pairs are added. $a_{i}$ and $a_{j}$ are dependent iff the joint MDD is empty, i.e., does not contain state $\left(g_{i}, g_{j}\right)$ at level $\max \left\{\mu_{i}, \mu_{j}\right\}$.

Figure 1(right) shows an example of merging the MDDs. The joint MDD starts at (B1, A2) at level 0. At level 1, we try all combinations of vertices at level 1 in both MDDs and add all of them to the joint MDD except for the pair (B2, B2), which represents a conflict space. We repeat this procedure at levels 2 and 3 until all branches of the joint MDD reach conflicting states and cannot be further developed. Therefore, in this example, the joint MDD is empty, and thus $a_{1}$ and $a_{2}$ are dependent.

\section{The WDG Heuristic}

For a CT node $N$ and two agents $a_{i}$ and $a_{j}$, we refer to the difference between the minimum sum of the costs of their conflict-free paths that satisfy N.constraints and the sum of the costs of their paths in N.solution as $\Delta_{i j}\left(\Delta_{i j} \geq 0\right) . a_{i}$ and $a_{j}$ are dependent iff $\Delta_{i j}>0$.

Although $G_{D}$ captures the information whether $\Delta_{i j}>0$ for any pair of agents, it does not capture the information how large the value of $\Delta_{i j}$ is. When $\Delta_{i j}>0$, the DG heuristic only uses 1 (a lower bound on $\Delta_{i j}$ ) as an admissible $h$-value. However, in some cases, $\Delta_{i j}$ could be larger than 1. For instance, in Figure 2, $\Delta_{12}=4$ because one of the agents must wait 4 timesteps at its start vertex. Therefore, we introduce the WDG heuristic, which captures not only the pairwise dependencies between agents but also the extra cost that each pair of dependent agents will contribute to the total cost.

\subsection{Weighted Pairwise Dependency Graph GWD}

We generalize the pairwise dependency graph to a weighted pairwise dependency graph $G_{W D}=\left(V_{D}, E_{D}, W_{D}\right)$ for $N$. It uses the same vertices and edges as $G_{D}$. The weight on each edge $\left(v_{i}, v_{j}\right) \in E_{D}$ equals $\Delta_{i j}$ at $N$. Here, $\Delta_{i j}$ is always larger than 0 as $a_{i}$ and $a_{j}$ are dependent. We also generalize the MVC to an edge-weighted minimum vertex cover (EWMVC), which is an assignment of non-negative integers $x_{1}, \ldots, x_{k}$, one for each vertex, which minimizes the sum of the $x_{i}$ subject to the constraints that $x_{i}+x_{j} \geq \Delta_{i j}$ for all $\left(v_{i}, v_{j}\right) \in E_{D} . x_{i}$ can be interpreted as the increase in the cost of the path of $a_{i}$. The sum of $x_{i}$ of the EWMVC of $G_{W D}$ is an admissible $h$-value for $N$ since, for each edge $\left(v_{i}, v_{j}\right) \in E_{D}$, the sum of the costs of the paths of agents $a_{i}$ and $a_{j}$ has to increase by at least $\Delta_{i j}$. We refer to this heuristic as the WDG heuristic. It strictly dominates the DG heuristic. Calculating the EWMVC is NP-hard since calculating the MVC is NP-hard and a special case of calculating EWMVC where the weights of all edges are 1. To calculate 
the EWMVC, we divide $G_{W D}$ into multiple connected components and calcualte the EWMVC on each component with a branch-and-bound algorithm that branches on the possible values of each $x_{i}$ in the component and prunes nodes using the cost of the best result so far. The EWMVC of $G_{W D}$ is the union of the EWMVCs of all components. Similar dependency graphs and EWMVCs for heuristic search were used in the context of MAPF for large agents [Li et al., 2019c], sliding tile puzzles [Felner et al., 2004] and cost-optimal planning [Pommerening et al., 2013].

\subsection{Constructing $\mathrm{G}_{W D}$}

We first construct the vertices and edges in $G_{W D}$ for $N$ using the same method as in Section 4. To calculate the weight $\Delta_{i j}$ for each edge $\left(v_{i}, v_{j}\right) \in E_{D}$, we run a MAPF algorithm to find the minimum sum of the costs of the conflict-free paths of $a_{i}$ and $a_{j}$ that satisfy N.constraints (ignoring the other agents). Here, the pathfinding problem is a two-agent problem with the constraints from N.constraints imposed on the two agents. Most optimal MAPF algorithms can be adapted to satisfy these constraints.

Similar to Section 4.2, for each non-root CT node, we need to find the edges and calculate the weights for only one agent (the one that has the new constraint) and can copy the rest of the edges and their weights from the parent CT node.

\subsection{The Two-Agent Problem}

We tried three search-based MAPF algorithms to solve the two-agent problem in our experiments: CBSH [Felner et al., 2018] (i.e., CBS with the CG heuristic), EPEA* [Goldenberg et al., 2014] and ICTS [Sharon et al., 2013], and CBSH is significantly faster than the other two.

One enhancement that we use in CBSH for the two-agent problem is that we set the $h$-value of the root CT node to 1. One is admissible because $\Delta_{i j}$ is at least 1 . This can help CBSH to resolve cardinal rectangle conflicts [Li et al., $2019 b$ ] or other symmetric conflicts efficiently. Figure 1(left) shows an example of a cardinal rectangle conflict. The cost of the optimal solution is 9. As CBSH searches in a best-first manner, it has to expand all CT nodes of cost 8 , even if it has already generated a CT node of cost 9 with an optimal solution. However, if the $h$-value of the root CT node is 1 , with a good tie-breaking rule (such as depth-first), CBSH can quickly generate a CT node of cost 9 with an optimal solution and return this solution immediately. In our experiments, this speeds up CBSH for the two-agent problem by up to 3 orders of magnitude.

\section{Runtime Reduction Techniques}

DG and WDG usually have larger $h$-values than CG. However, computing these heuristics incurs overhead per CT node. In this section, we introduce a number of simple techniques to reduce the runtime overhead for the calculation of the heuristics.

Lazy Computation of Heuristics. The high-level search of CBSH resembles an $A^{*}$ search, so techniques to speed up A* can also be applied here. Lazy A* [Tolpin et al., 2013] improves $A^{*}$ by evaluating expensive heuristics lazily. Instead of computing the expensive $h$-value $h_{2}(N)$ immediately after generating a new node $N$, lazy $\mathrm{A}^{*}$ first computes a cheaper but less informed $h$-value $h_{1}(N)$ (or even uses zero) and inserts $N$ into OPEN. Only when $N$ emerges from OPEN, it computes $h_{2}(N)$ for it and re-inserts it into OPEN.

Here, for simplicity, we view both the conflict graph and the pairwise dependency graph as an edge-weighted pairwise dependency graph whose edges all have weight one. Each of the CG, DG or WDG heuristics is treated as $h_{2}$, and we define $h_{1}$ for a child CT node $N^{\prime}$ of $N$ as $\max \{N . h-$ $\max _{j:(i, j) \in E_{D}} \Delta_{i j}, N$. cost $+N . h-N^{\prime}$. cost, 0$\}$, where $i$ is the index of the agent whose path gets re-planned at $N^{\prime}$. The first term $N . h-\max _{j:(i, j) \in E_{D}} \Delta_{i j}$ is a lower bound on the sum of $x_{i}$ of the EWMVC of the sub-graph of $G_{W D}$ of $N$ without edges incident on vertex $v_{i}$. It is admissible because the sum of $x_{i}$ of the EWMVC of $G_{W D}$ of $N^{\prime}$ should be no smaller than the sum of $x_{i}$ of the EWMVC of this sub-graph. The second term $N$.cost $+N . h-N^{\prime}$.cost is admissible because the $f$-value (i.e., N.cost $+N$.h) is non-decreasing. Empirically, the runtime overhead of OPEN operations (e.g., insert or pop a node) is negligible.

Memoization. Memoization is an optimization technique to speed up algorithms by caching the results of expensive function calls and returning the cached results when the same inputs occur again. Here, we use memoization to store the results of merging the MDDs and solving the two-agent problems. The inputs are the indices of two agents and the set of constraints imposed on them. The output is the existence of the corresponding edge and, if its exists, its edge weight. Empirically, the memory overhead of caching and the runtime overhead of storing and retrieving results are both negligible, and the cached results are used frequently. This is because CBS often repeatedly resolves the same conflict in different branches, and many CT nodes thus have the same set of constraints imposed on the same agent. Memoization can also be used to save runtime for building MDDs.

\section{Experimental Results}

We experiment with CBS, ICBS and CBSH with the CG, DG and WDG heuristics on 4-neighbor grids. All CBSH solvers use the two improvements discussed in Section 6, and the WDG heuristic uses the CBSH algorithm discussed in Section 5.3 to solve the two-agent problem. We generate 50 instances with random start and goal vertices for each map and each number of agents. Our code is written in $\mathrm{C}++$, and our experiments are conducted on a $2.80 \mathrm{GHz}$ Intel Core i7-7700 laptop with 8 GB RAM.

\subsection{Small Maps}

First, we test the solvers on $20 \times 20$ grids. We focus on an empty map, which is a $20 \times 20$ grid with no blocked cells, and a dense map, which is a $20 \times 20$ grid with $30 \%$ randomly blocked cells. We use a time limit of 1 minute for each solver on each instance.

$h$-values of the root CT node. Table 1 shows the $h$-values of the root CT node. On the empty map, DG is much larger than CG while WDG is only slightly larger than DG because agents on the empty map usually have many bypasses, and 
Proceedings of the Twenty-Eighth International Joint Conference on Artificial Intelligence (IJCAI-19)

\begin{tabular}{|c|ccr||c|ccc||cccr|}
\hline \multicolumn{3}{|c||}{ Empty map } & \multicolumn{4}{c||}{ Dense map } & \multicolumn{4}{c|}{ 20 agents } \\
\hline$k$ & CG & DG & WDG & $k$ & CG & DG & WDG & obs & CG & DG & WDG \\
\hline 30 & 0.2 & 1.0 & 1.2 & 16 & 3.9 & 3.9 & 11.6 & 0 & 0.1 & 0.5 & 0.5 \\
40 & 0.5 & 1.7 & 2.0 & 20 & 4.8 & 4.8 & 15.2 & 10 & 1.0 & 1.3 & 2.1 \\
50 & 0.6 & 2.3 & 2.8 & 24 & 6.9 & 7.0 & 22.2 & 20 & 3.0 & 3.1 & 6.2 \\
\hline
\end{tabular}

Table 1: Average $h$-values of the root CT node. $k$ represents the number of agents, and obs represents the percentage of cells that are randomly blocked on a $20 \times 20$ grid.

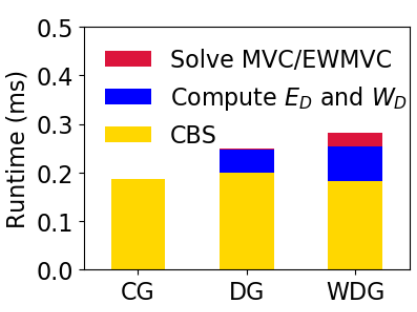

(a) Empty map.

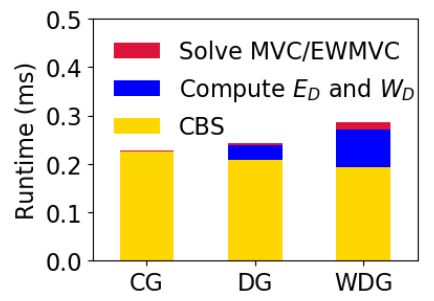

(b) Dense map.
Figure 3: Average runtime per expanded CT node over 300 instances with different numbers of agents.

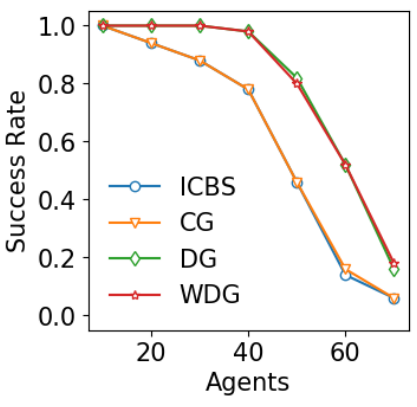

(a) Empty map.

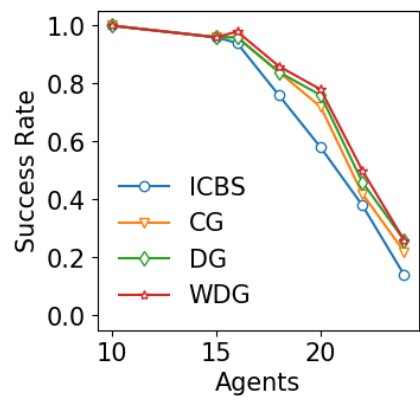

(b) Dense map.
Figure 4: Success rate (i.e., the percentage of solved instances).

thus $\Delta_{i j}$ is 0 or 1 in most cases. However, on the dense map, DG is only slightly larger than CG while WDG is much larger than both of them because most conflicts are cardinal and the map contains many narrow corridors, which induce a large $\Delta_{i j}$. The last four columns show the results for 20 agents on grids with increasing obstacle densities to provide more details on the transition from empty grids to dense grids.

Runtime overhead of heuristics calculation. Figure 3 shows the runtime breakdown per CT node. The CBS runtimes (yellow) of the three solvers are slightly different because the different heuristics cause CBS to expand different sets of CT nodes. The runtimes of constructing $G_{D}$ and $G_{W D}$ (blue) are small due to the memoization technique, which saves more than $90 \%$ of the edge and weight computation time. Although we use simple algorithms to solve the NPhard problems MVC and EWMVC, their runtimes (red) are also small due to the small sizes of $G_{D}$ and $G_{W D}$. The lazy computation of heuristics also contributes to the reduction in the runtime overhead as the expensive heuristics are computed for only $65 \%$ of the generated CT nodes.

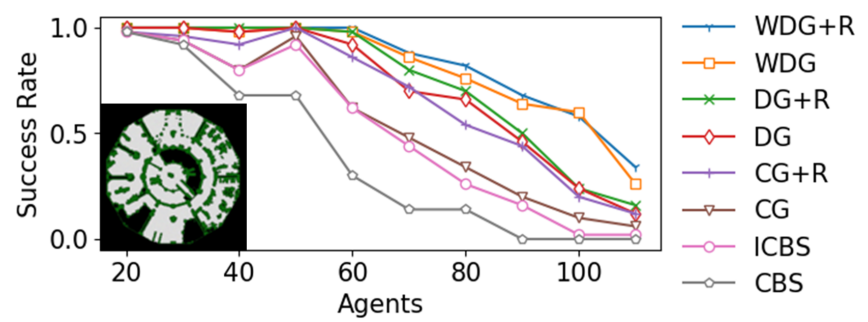

Figure 5: Success rate on on the large map. "+R" means that the solver uses the rectangle reasoning technique.

Overall performance. Figure 4 and Table 2 show the success rate, the average number of expanded CT nodes and the average runtime of the solvers. The number of expanded CT nodes is consistent with the computed $h$-value of the root CT node. That is, a larger $h$-value usually leads to a smaller number of expanded CT nodes. With respect to success rate and runtime, both DG and WDG outperform CG. In particular, $D G$ runs slightly faster than WDG on the empty map as it has a smaller runtime overhead than WDG, while WDG runs much faster than $D G$ on the dense map as it leads to a larger node reduction than $D G$. The usefulness of $C G$ heavily depends on the particular instance. It has almost the same performance as ICBS (= CBSH with $h$-values that are always zero) on the empty map where cardinal conflicts are rare. However, our new heuristics are efficient on both maps and up to 9 times faster than CG (e.g., for 40 agents on the empty map) for the solved instances (which are relatively easy).

\subsection{Large Maps}

Next, we test the solvers on a large map. We use the benchmark game map lak503d from [Sturtevant, 2012], which is a $192 \times 192$ grid with 51\% blocked cells (Figure 5).

Plugging in the rectangle reasoning technique. $\mathrm{Li}$ et $a l$. [2019b] analyze the symmetries in grid-based MAPF and improve $\mathrm{CBSH}$ using a rectangle reasoning technique. This technique is able to find some (but not all) edges in $G_{D}$, so it can save some of the effort of merging the MDDs. Therefore, we add the most advanced rectangle reasoning technique RM from [Li et al., 2019b] to all CBSH solvers. Figure 5 shows their success rates within 1 minute. In particular, $C G+R$ is identical to CBSH-RM from [Li et al., 2019b] and is the state-of-the-art CBS-based solver in previous research. The results show that all new solvers beat the previous solvers. The rectangle reasoning technique slightly speeds up WDG in most cases. As a result, $\mathrm{WDG}+\mathrm{R}$ is the strongest solver among them and therefore is the new state-of-the-art CBSbased solver.

Results with longer time limits. Figure 6(left) shows the success rates over 50 random instances of 100 agents on the large map with different time limits. As the time limit increases, the benefit of using WDG and DG over CG increases as well. In general, it is worth spending some extra time per CT node to obtain a better $h$-value, since a larger $h$-value usually leads to an exponential reduction in the number of CT nodes. Figure 6(right) shows the results with a time limit of 30 minutes. Although DG and WDG have a larger runtime 
Proceedings of the Twenty-Eighth International Joint Conference on Artificial Intelligence (IJCAI-19)

\begin{tabular}{|c|r|rrrr|}
\hline Agents & Instances & \multicolumn{1}{|c|}{ ICBS } & CG & DG & WDG \\
\hline \multicolumn{7}{|c|}{ Nodes $(\times 1000)$} \\
\hline 30 & 44 & 3.6 & 2.6 & $\mathbf{0 . 5}$ & $\mathbf{0 . 5}$ \\
40 & 39 & 8.9 & 7.0 & $\mathbf{0 . 2}$ & $\mathbf{0 . 2}$ \\
50 & 23 & 12.4 & 10.1 & $\mathbf{2 . 9}$ & $\mathbf{2 . 9}$ \\
\hline \multicolumn{7}{|c|}{ Runtime (s) } \\
\hline 30 & 44 & 0.5 & 0.4 & $\mathbf{0 . 1}$ & $\mathbf{0 . 1}$ \\
40 & 39 & 1.0 & 0.9 & $\mathbf{0 . 1}$ & $\mathbf{0 . 1}$ \\
50 & 23 & 1.7 & 1.5 & $\mathbf{0 . 6}$ & 0.7 \\
\hline
\end{tabular}

(a) Empty map.

\begin{tabular}{|c|c|rrrr|}
\hline Agents & Instances & \multicolumn{1}{c|}{ ICBS } & \multicolumn{1}{c|}{ CG } & DG & WDG \\
\hline \multicolumn{7}{|c|}{ Nodes $(\times 1000)$} \\
\hline 16 & 47 & 20.2 & 9.6 & 7.8 & $\mathbf{6 . 1}$ \\
20 & 29 & 20.2 & 13.6 & 10.7 & $\mathbf{8 . 9}$ \\
24 & 7 & 79.6 & 47.4 & 33.2 & $\mathbf{1 5 . 2}$ \\
\hline \multicolumn{7}{|c|}{ Runtime (s) } \\
\hline 16 & 47 & 7.0 & $\mathbf{2 . 4}$ & $\mathbf{2 . 4}$ & $\mathbf{2 . 4}$ \\
20 & 29 & 4.0 & 3.3 & 2.1 & $\mathbf{1 . 9}$ \\
24 & 7 & 17.9 & 9.6 & 5.4 & $\mathbf{3 . 0}$ \\
\hline
\end{tabular}

(b) Dense map.

Table 2: Average expanded CT nodes and average runtime over instances solved by all solvers.

\begin{tabular}{|c|c|c|c|c|c|c|c|c|c|c|c|c|c|c|}
\hline \multicolumn{5}{|c|}{ Empty map } & \multicolumn{5}{|c|}{ Dense map } & \multicolumn{5}{|c|}{ Large map } \\
\hline Agents & $\mathrm{CG}$ & DG & WDG & $h^{*}$ & Agents & CG & $\mathrm{DG}$ & WDG & $h^{*}$ & Agents & $C G$ & DG & WDG & $h^{*}$ \\
\hline 30 & 0.2 & 1.0 & 1.2 & 1.7 & 16 & 3.9 & 3.9 & 11.5 & 18.6 & 60 & 3.6 & 4.0 & 6.7 & 7.6 \\
\hline 40 & 0.5 & 1.6 & 2.0 & 3.3 & 20 & 4.7 & 4.7 & 14.0 & 23.2 & 80 & 5.7 & 6.5 & 10.9 & 12.2 \\
\hline 50 & 0.5 & 2.2 & 2.6 & 4.7 & 24 & 6.5 & 6.5 & 18.9 & 28.5 & 100 & 8.6 & 9.2 & 15.6 & 18.0 \\
\hline
\end{tabular}

Table 3: Average $h$-values and average $h^{*}$-values of the root CT node over instances of which the $h^{*}$-value is known, i.e., instances solved by at least one CBSH solver.

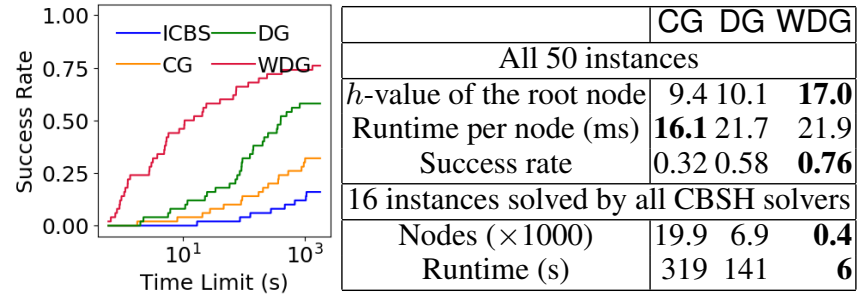

Figure 6: Results for 100 agents on the large map. The right table uses a time limit of 30 minutes.

overhead compared to small maps, WDG significantly outperforms DG, which - in turn - significantly outperforms CG in terms of both success rate and runtime. For example, compared with CG, WDG improves the success rate by a factor of 2 and runs faster by a factor of 50 .

\subsection{Comparing with the Perfect Heuristic}

Table 3 compares the average $h$-values of the root CT node by different CBSH solvers with the average $h^{*}$-values of the root CT node (i.e., the optimal solution cost minus the cost of the root CT node). On the dense map, WDG is significantly smaller than $h^{*}$ because agents are deeply coupled and reasoning about the pairwise dependencies between agents is not enough. However, on the empty map or the large map, WDG is close to $h^{*}$ because agents are less coupled and reasoning about the pairwise dependencies between agents is enough in many cases. In other words, $h / h^{*}$ is closer to 1 on the empty map or the large map than on the dense map. This explains why, compared to CG, WDG has the largest $h$-value improvement on the dense map over all three maps (as shown in Table 1) but the smallest node reduction factor on the dense map over all three maps (as shown in Table 2 and Figure 6).

\section{Conclusions and Future Work}

In this paper, we analyzed the limitations of the heuristic used to provide high-level guidance for CBS, a state-of-theart algorithm for multi-agent path finding. We proposed two new admissible heuristics by reasoning about the pairwise dependencies between agents. They always dominate the old heuristic and only incur a small runtime overhead per node. Empirically, they increase the success rates and speeds of CBS with the old heuristic by up to a factor of 50 .

There are several interesting directions for future work. (1) Study admissible or inadmissible heuristics for sub-optimal CBS-based algorithms [Barer et al., 2014]. (2) Apply similar heuristics to other MAPF algorithms, such as ICTS [Sharon et al., 2013] or MDD-SAT [Surynek et al., 2016]. (3) Generalize these heuristics to groups larger than pairs of agents, e.g., to triples and quadruples.

\section{Acknowledgments}

The research at the University of Southern California was supported by the National Science Foundation (NSF) under grant numbers 1409987, 1724392, 1817189 and 1837779 as well as a gift from Amazon. The research was also supported by the United States-Israel Binational Science Foundation (BSF) under grant number 2017692.

\section{A Proof of Theorem 1}

In order to help explain the proof, we define another type of MDDs, called extended MDDs, that ignore constraints, i.e., they are allowed to include nodes prohibited by constraints. Formally, an extended MDD $M D D_{i}^{\mu}$ for $a_{i}$ is a directed acyclic graph that consists of all paths of $a_{i}$ from $s_{i}$ to $g_{i}$ within $\mu$ timesteps. In particular, $M D D_{i}^{\mu}$ is empty iff $\mu$ is smaller than the minimum cost of the path of $a_{i}$. All MDDs we discuss in this section are extended MDDs. We say that a node $(x, t) \in M D D_{i}^{\mu}$ iff $M D D_{i}^{\mu}$ has vertex $x$ at level $t$, i.e., there is a path from $s_{i}$ at timestep 0 to $x$ at timestep $t$ 

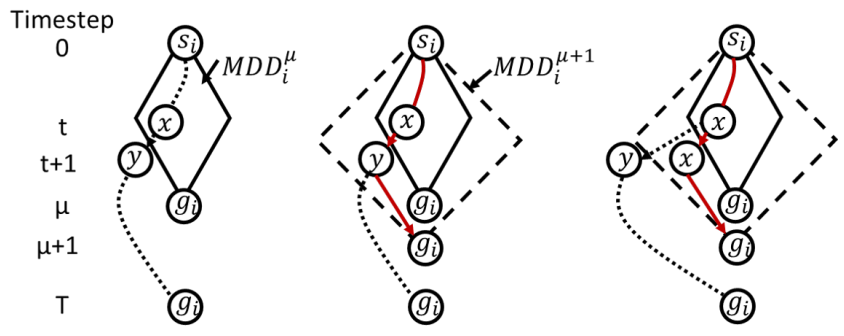

Figure 7: Constructing a path of cost $\mu+1$. The solid and dashed diamonds include all nodes in $M D D_{i}^{\mu}$ and $M D D_{i}^{\mu+1}$, respectively.

and then to $g_{i}$ by timestep $\mu$. Let $\operatorname{dist}(x, y)$ denote the distance between vertices $x$ and $y$ and $P(t)$ denote the vertex at timestep $t$ on path $P$. Then, the extended MDDs have the following two properties:

(1) $(x, t) \in M D D_{i}^{\mu}$ iff $\operatorname{dist}\left(s_{i}, x\right) \leq t$ and $\operatorname{dist}\left(x, g_{i}\right) \leq$ $\mu-t$.

(2) For any path $P$ of $a_{i}$, if $(P(t), t) \notin M D D_{i}^{\mu}$, then $\left.\left(P\left(t^{\prime}\right)\right), t^{\prime}\right) \notin M D D_{i}^{\mu}$ for all $t^{\prime} \geq t$.

Property (1) can be obtained from the definition. Property (2) holds because, by contradiction, if $\left.\left(P\left(t^{\prime}\right)\right), t^{\prime}\right) \in M D D_{i}^{\mu}$, then there is a sub-path $P^{\prime}$ in $M D D_{i}^{\mu}$ from $P\left(t^{\prime}\right)$ at timestep $t^{\prime}$ to $g_{i}$ at timestep $\mu$. The path that follows a prefix of $P$ from $s_{i}$ at timestep 0 to $P\left(t^{\prime}\right)$ at timestep $t^{\prime}$ and then follows $P^{\prime}$ to $g_{i}$ at timestep $\mu$ traverses $P(t)$ at timestep $t$, i.e., $(P(t), t) \in M D D_{i}^{\mu}$. Property (2) tells us that, once a path leaves an extended MDD, it will never revisit this extended MDD later.

We say that $M D D_{i}^{\mu}$ includes all nodes prohibited by a set of constraints $C$ iff $M D D_{i}^{\mu}$ has vertex $v$ at level $t$ for any vertex constraint $\left\langle a_{i}, v, t\right\rangle \in C$ and edge $(u, v)$ from level $t$ to level $t+1$ for any edge constraint $\left\langle a_{i}, u, v, t\right\rangle \in C$.

Lemma 2. Let $C$ be a set of constraints and $\mu$ be a large enough integer such that $M D D_{i}^{\mu}$ is not empty and includes all nodes prohibited by $C$. If $a_{i}$ has a path that satisfies $C$, then $a_{i}$ has a path of cost no more than $\mu+1$ that satisfies $C$.

Proof. By assumption, $a_{i}$ has a path $P$ that satisfies $C$. Let $T$ be the cost of $P$. If $T \leq \mu+1$, then we are done. Otherwise, as shown in Figure 7, we will construct a path of cost $\mu+1$ by letting $a_{i}$ (1) first follow the prefix of $P$ to the border of $M D D_{i}^{\mu}$, (2) then cross the border to a node in $M D D_{i}^{\mu+1}$, and (3) last follow a path in $M D D_{i}^{\mu+1}$ to $g_{i}$ at timestep $\mu+1$. This new path satisfies $C$ because $P$ satisfies $C$ and any nodes or edges outside $M D D_{i}^{\mu}$ also satisfy $C$. The existence of such a path is proved as follows.

Node $\left(s_{i}, 0\right)$ (the first node of $P$ ) is in $M D D_{i}^{\mu}$. Node $\left(g_{i}, T\right)$ (the last node of $P$ ) is not in $M D D_{i}^{\mu}$ (because $T>\mu$ ). So, there is a timestep $t<T$ such that $(P(t), t) \in M D D_{i}^{\mu}$ and $(P(t+1), t+1) \notin M D D_{i}^{\mu}$. Let $x$ and $y$ represent $P(t)$ and $P(t+1)$, respectively (Figure 7(left)). There are now two cases, and we show how to construct a path of cost $\mu+1$ that satisfies $C$ in each case.

Case 1: $(y, t+1) \in M D D_{i}^{\mu+1}$ (Figure 7(middle)). There is a sub-path $P^{\prime}$ in $M D D_{i}^{\mu+1}$ from $y$ at timestep $t+1$ to $g_{i}$ at timestep $\mu+1$. By applying $(y, t+1) \notin M D D_{i}^{\mu}$ to Property (2), $P^{\prime}$ does not traverse any MDD node in $M D D_{i}^{\mu}$, and thus does not violate any constraints. Therefore, a path that follows a prefix of $P$ from $s_{i}$ at timestep 0 to $y$ at timestep $t+1$ and then follows $P^{\prime}$ to $g_{i}$ at timestep $\mu+1$ is a path of cost $\mu+1$ that satisfies $C$.

Case 2: $(y, t+1) \notin M D D_{i}^{\mu+1}$ (Figure 7(right)). Since node $(y, t+1)$ is on $P$, $\operatorname{dist}\left(s_{i}, y\right) \leq t+1$. Then, from Property (1), dist $\left(y, g_{i}\right)>(\mu+1)-(t+1)$. Rearranging the terms yields

$$
\begin{aligned}
\mu & \leq t+\operatorname{dist}\left(y, g_{i}\right)-1 \\
& \leq t+\operatorname{dist}(y, x)+\operatorname{dist}\left(x, g_{i}\right)-1 \\
& \leq t+\operatorname{dist}\left(x, g_{i}\right) \\
& \leq \mu .
\end{aligned}
$$

Inequality (2) is based on the the triangle inequality. Inequality (3) is from $\operatorname{dist}(y, x) \leq 1$. Inequality (4) is obtained by applying $(x, t) \in M D D_{i}^{\mu}$ to Property (1). By comparing the first line and the last line of these inequalities, all lines are actually equal. Specifically, for Inequality (4),

$$
t+\operatorname{dist}\left(x, g_{i}\right)=\mu .
$$

Since node $(x, t)$ is on $P$, dist $\left(s_{i}, x\right) \leq t$. From Equation (5) and Property (1), we know that $(x, t+1) \notin M D D_{i}^{\mu}$ and $(x, t+1) \in M D D_{i}^{\mu+1}$. Hence, there is a sub-path $P^{\prime \prime}$ in $M D D_{i}^{\mu+1}$ from $x$ at timestep $t+1$ to $g_{i}$ at timestep $\mu+1$. From Property (2), $P^{\prime \prime}$ does not traverse any MDD node in $M D D_{i}^{\mu}$, and thus does not violate any constraints. Therefore, a path that follows a prefix of $P$ from $s_{i}$ at timestep 0 to $x$ at timestep $t$, waits for one timestep and then follows $P^{\prime \prime}$ to $g_{i}$ at timestep $\mu+1$ is a path of cost $\mu+1$ that satisfies $C$.

Finally, we prove Theorem 1 based on Lemma 2.

Proof. Without loss of generality, we only focus on $N_{1}$. Every constraint in N.constraints imposed on $a_{i}$ was generated due to a conflict that occurred on one of the old paths of $a_{i}$, i.e., paths of $a_{i}$ in the solutions of any ancestor CT nodes of $N$. The cost of any old path of $a_{i}$ is no larger than $\mu_{i}$. Therefore, $M D D_{i}^{\mu_{i}}$ includes all nodes prohibited by N.constraints.

If the chosen conflict occurs before $a_{i}$ reaches $g_{i}$ (i.e., $t<\mu_{i}$ ), then $M D D_{i}^{\mu_{i}}$ includes all nodes prohibited by $N_{1}$.constraints. From Lemma 2, we know that $a_{i}$ has a path of cost no more than $\mu_{i}+1$ that satisfies $N_{1}$.constraints. So, the cost-minimal path of $a_{i}$ is of cost at most $\mu_{i}+1$, and $N_{1}$. cost $\in\{N$. cost,$N . \cos t+1\}$.

If the chosen conflict occurs after $a_{i}$ reaches $g_{i}$ (i.e., $t \geq$ $\left.\mu_{i}\right)$, then the chosen conflict is $\left\langle a_{i}, a_{j}, g_{i}, t\right\rangle$ and the additional constraint added to $N_{1}$ is $\left\langle a_{i}, g_{i}, t\right\rangle . M D D_{i}^{t}$ includes all nodes prohibited by $N_{1}$.constraints, and thus, by Lemma 2, $a_{i}$ has a path of cost no more than $t+1$ that satisfies $N_{1}$.constraints. On the other hand, since $a_{i}$ is prohibited from being at $g_{i}$ at timestep $t$, its cost-minimal path is of cost at least $t+1$. Therefore, the cost-minimal path of $a_{i}$ is of cost $t+1$, and $N_{1} \cdot \cos t=N \cdot \cos t+t+1-\mu_{i}$. 


\section{References}

[Barer et al., 2014] Max Barer, Guni Sharon, Roni Stern, and Ariel Felner. Suboptimal variants of the conflict-based search algorithm for the multi-agent pathfinding problem. In SoCS, pages 19-27, 2014.

[Barták et al., 2017] Roman Barták, Jirı Švancara, and Marek Vlk. Scheduling models for multi-agent path finding. In MISTA, pages 189-200, 2017.

[Boyarski et al., 2015] Eli Boyarski, Ariel Felner, Roni Stern, Guni Sharon, David Tolpin, Oded Betzalel, and Solomon Eyal Shimony. ICBS: Improved conflict-based search algorithm for multi-agent pathfinding. In IJCAI, pages 740-746, 2015.

[Cohen et al., 2016] Liron Cohen, Tansel Uras, T. K. Satish Kumar, Hong Xu, Nora Ayanian, and Sven Koenig. Improved solvers for bounded-suboptimal multi-agent path finding. In IJCAI, pages 3067-3074, 2016.

[Cohen et al., 2018] Liron Cohen, Glenn Wagner, David M. Chan, Howie Choset, Nathan Sturtevant, Sven Koenig, and T. K. Satish Kumar. Rapid randomized restarts for multiagent path finding solvers. In SoCS, pages 1909-1911, 2018.

[Erdem et al., 2013] Esra Erdem, Doga Gizem Kisa, Umut Öztok, and Peter Schüller. A general formal framework for pathfinding problems with multiple agents. In $A A A I$, pages 290-296, 2013.

[Felner et al., 2004] Ariel Felner, Richard E. Korf, and Sarit Hanan. Additive pattern database heuristics. Journal of Artificial Intelligence Research, 22:279-318, 2004.

[Felner et al., 2018] Ariel Felner, Jiaoyang Li, Eli Boyarski, Hang Ma, Liron Cohen, T. K. Satish Kumar, and Sven Keonig. Adding heuristics to conflict-based search for multi-agent path finding. In ICAPS, pages 83-87, 2018.

[Gange et al., 2019] Graeme Gange, Daniel Harabor, and Peter J. Stuckey. Lazy CBS: Implicit conflict-based search using lazy clause generation. In ICAPS, 2019.

[Goldenberg et al., 2014] Meir Goldenberg, Ariel Felner, Roni Stern, Guni Sharon, Nathan R. Sturtevant, Robert C. Holte, and Jonathan Schaeffer. Enhanced partial expansion A*. Journal of Artificial Intelligence Research, 50:141187, 2014.

[Hönig et al., 2018] Wolfgang Hönig, James A. Preiss, T. K. Satish Kumar, Gaurav S. Sukhatme, and Nora Ayanian. Trajectory planning for quadrotor swarms. IEEE Transactions on Robotics, 34(4):856-869, 2018.

[Hönig et al., 2019] Wolfgang Hönig, Scott Kiesel, Andrew Tinka, Joseph W. Durham, and Nora Ayanian. Persistent and robust execution of MAPF schedules in warehouses. IEEE Robotics and Automation Letters, 4(2):1125-1131, 2019.

[Li et al., 2019a] Jiaoyang Li, Daniel Harabor, Peter J. Stuckey, Ariel Felner, Hang Ma, and Sven Keonig. Disjoint splitting for multi-agent path finding with conflictbased search. In ICAPS, 2019.
[Li et al., 2019b] Jiaoyang Li, Daniel Harabor, Peter J. Stuckey, Hang Ma, and Sven Koenig. Symmetry-breaking constraints for grid-based multi-agent path finding. In AAAI, 2019.

[Li et al., 2019c] Jiaoyang Li, Pavel Surynek, Ariel Felner, Hang Ma, T. K. Satish Kumar, and Sven Koenig. Multiagent path finding for large agents. In $A A A I, 2019$.

[Ma et al., 2017a] Hang Ma, Jiaoyang Li, T. K. Satish Kumar, and Sven Koenig. Lifelong multi-agent path finding for online pickup and delivery tasks. In $A A M A S$, pages 837-845, 2017.

[Ma et al., 2017b] Hang Ma, Jingxing Yang, Liron Cohen, T. K. Satish Kumar, and Sven Koenig. Feasibility study: Moving non-homogeneous teams in congested video game environments. In AIIDE, pages 270-272, 2017.

[Pommerening et al., 2013] Florian Pommerening, Gabriele Röger, and Malte Helmert. Getting the most out of pattern databases for classical planning. In IJCAI, pages 23572364, 2013.

[Sharon et al., 2013] Guni Sharon, Roni Stern, Meir Goldenberg, and Ariel Felner. The increasing cost tree search for optimal multi-agent pathfinding. Artificial Intelligence, 195:470-495, 2013.

[Sharon et al., 2015] Guni Sharon, Roni Stern, Ariel Felner, and Nathan R. Sturtevant. Conflict-based search for optimal multi-agent pathfinding. Artificial Intelligence, 219:40-66, 2015.

[Silver, 2005] David Silver. Cooperative pathfinding. In AIIDE, pages 117-122, 2005.

[Standley, 2010] Trevor Scott Standley. Finding optimal solutions to cooperative pathfinding problems. In $A A A I$, pages 173-178, 2010.

[Sturtevant, 2012] Nathan R. Sturtevant. Benchmarks for grid-based pathfinding. Transactions on Computational Intelligence and AI in Games, 4(2):144-148, 2012.

[Surynek et al., 2016] Pavel Surynek, Ariel Felner, Roni Stern, and Eli Boyarski. Efficient SAT approach to multiagent path finding under the sum of costs objective. In ECAI, pages 810-818, 2016.

[Tolpin et al., 2013] David Tolpin, Tal Beja, Solomon Eyal Shimony, Ariel Felner, and Erez Karpas. Toward rational deployment of multiple heuristics in $\mathrm{A}^{*}$. In IJCAI, pages 674-680, 2013.

[Wagner and Choset, 2011] Glenn Wagner and Howie Choset. $\mathrm{M}^{*}$ : A complete multirobot path planning algorithm with performance bounds. In IROS, pages 3260-3267, 2011.

[Yu and LaValle, 2013a] Jingjin Yu and Steven M. LaValle. Planning optimal paths for multiple robots on graphs. In ICRA, pages 3612-3617, 2013.

[Yu and LaValle, 2013b] Jingjin Yu and Steven M. LaValle. Structure and intractability of optimal multi-robot path planning on graphs. In $A A A I$, pages 1444-1449, 2013. 